\title{
Cosmopolitismo Feminista contra Globalización
}

\section{Feminist Cosmopolitanism against Globalization}

\author{
Sonia Reverter-Bañón ${ }^{1}$ \\ Universitat Jaume I (España)
}

Recibido: 31-08-16

Aprobado: 06-03-17

\section{Resumen}

Si bien los conceptos de globalización y cosmopolitismo se suelen entender como teóricamente relacionados o co-implicados, en la práctica actual constatamos que no es así. La idea que mantenemos es que el actual proceso de globalización en su forma neoliberal, y apuntalada por organismos internacionales como el Banco Mundial, impide un cosmopolitismo que pueda avanzar la agenda de igualdad a nivel global. La propuesta que presentamos de un cosmopolitismo feminista pretende servir de crítica fundamental a esa manera de entender y practicar la globalización. El reto para nuestra propuesta será el de poder conformar un sujeto cosmopolita que buscando esa igualdad de género supere, a su vez, tanto un universalismo formal saturado de un sujeto patriarcal, como un discurso de las diferencias que no logra fraguar un sujeto político que no se fragmente en las diferentes luchas necesarias para una igualdad global.

Palabras-clave: igualdad de género, feminismo, globalización, cosmopolitismo, universalismo, diferencias.

\footnotetext{
${ }^{1}$ (reverter@uji.es) Doctora en Filosofía. Profesora Titular de Filosofía en la Universitat Jaume I. Imparte clases de filosofía contemporánea y de teoría feminista. Miembro fundador del Instituto Universitario de Estudios Feministas y de Género de la UJI. Vicedecana para la dirección del grado de "Humanidades. Estudios Interculturales". En relación a la temática presentada en este artículo ha publicado principalmente: Civil Society and Gender Equality: A Theoretical Approach, en Civil Society Series (2006, London School of Economics and Political Sciences). Y "Género y Gobernanza", en González-Esteban, Elsa, ed. Ética y Gobernanza. Un cosmopolitismo para el siglo XXI, (2013, Comares). El último artículo publicado es "Performatividad: la teoría especial y la general", Isegoría, 2017. Forma parte del equipo de investigadores/as del Proyecto de Investigación I+D+I del Ministerio de Economía y Competitividad FFI2016-76753-C2-2-P.
} 


\begin{abstract}
While the concepts of globalization and cosmopolitanism are often seen as theoretically co-related, in current practice, we perceive that it is not so. In our paper we maintain that the current process of globalization in its neoliberal form, buttressed by international organizations such as the World Bank, prevents a cosmopolitanism that could globally advance the equality agenda. Our proposal of a feminist critical cosmopolitanism is intended as a criticism of the way of understanding and practicing globalization. The challenge for our proposal will be to shape a cosmopolitan subject that seeking gender equality could exceed, in turn, both formal universalism saturated with a patriarchal subject, and a discourse of differences that fails to forge a political subject, capable of moving in the different struggles needed for global equality without fragmenting.
\end{abstract}

Key-words: Gender Equality, Feminism, Globalization, Cosmopolitanism, Universalism, Differences.

\title{
1. Introducción
}

$\mathrm{Si}$ bien las organizaciones internacionales suelen afirmar que la globalización ha mejorado la igualdad entre mujeres y hombres (Potrafke 2015), no es menos cierto que los datos mundiales constatan que ha empeorado la justicia social y económica de muchos (Milanovic 2016). Desde nuestra perspectiva esto nos resulta contradictorio, y alienta una interpretación, que no compartimos, en la línea de dividir o separar la justicia social de la igualdad. Es decir, y según esta interpretación, se es igual en injusticia. O se es más igual en un mundo más injusto, lo cual acaba estableciendo la igualdad entre géneros en un mero trámite formal y no real. Ello nos lleva a la necesidad de analizar dos conceptos que usualmente se entienden como correlativos o co-implicados: globalización (como proceso de integración internacional) y cosmopolitismo (como teoría que implica la pertenencia de todos los seres humanos a una comunidad común).

Nuestra tesis es que en la actualidad los términos de globalización y cosmopolitismo no sólo no se co-implican, sino que, como veremos, el primero está yendo contra el segundo (Barbalet 2014). Una globalización que atenta contra el cosmopolitismo significa, esta es nuestra tesis, una seria amenaza para la igualdad. Por ello, la propuesta que aquí formulamos trata de enunciar un cosmopolitismo que pueda hacer frente a una globalización que pone en peligro los progresos en igualdad de género. Este cosmopolitismo que hemos denominado "feminista" habrá de superar, sin embargo, un reto importante: 
no dejarse cooptar por la agenda de la globalización neoliberal. El reto será, así, construir un cosmopolitismo que no pueda ser cooptado y que, a su vez, pueda articular un sujeto cosmopolita que sea capaz de resolver las tensiones entre globalización e igualdad entre mujeres y hombres. Para ello articularemos nuestra propuesta en tres apartados.

-En primer lugar (apartado 2) analizaremos quién es el sujeto real en el proceso de globalización actual y quién es el sujeto de las políticas de igualdad. Al hacer este análisis tendremos que rechazar tanto la propuesta del sujeto universal (por falsa y patriarcal) como la propuesta de un sujeto que está tan lleno de diferencias y fragmentaciones que se vuelve imposible pensarlo en una unidad, dejándonos sin el sujeto "las mujeres".

-En segundo lugar (apartado 3), y a modo de ejemplo, observaremos la agenda de igualdad mantenida por instituciones internacionales, como el Banco Mundial, criticando cómo en sus intereses neoliberales y desde la llamada "Smart Economics" proponen una visión esencialista de las mujeres que retiene a éstas en su experiencia de cuidadoras, propagando con ello una limitada y pobre comprensión de la igualdad de género.

-En tercer lugar (apartado 4) propondremos la necesidad de un cosmopolitismo, que podemos denominar "cosmopolitismo feminista", que pueda tanto superar esta limitación como enfrentar una globalización que se está llevando a cabo sin un progreso real en la agenda de igualdad.

-En cuarto lugar (apartado 5) concluiremos cómo vertebrar ese cosmopolitismo feminista como práctica política emancipatoria que pueda anular los efectos perversos de la globalización neoliberal, y a la vez abrir y ampliar el marco de gobernanza actual.

\section{El sujeto político y su identidad sexo/género: ¿universal o particular?}

La relación entre globalización e igualdad de género es una de carácter complejo. Si bien la internacionalización de instituciones ha podido conllevar innegables mejoras para la igualdad de mujeres y hombres, el desarrollo económico que conlleva la globalización se está llevando a cabo deteriorando la visión cosmopolita que teóricamente debería estar asociada a esa globalización, y ello a su vez tiene implicaciones nefastas para la realidad igualitaria entre los géneros.

La agenda de igualdad para las mujeres ha ido promoviendo cambios continuos hacia una mayor globalización. Si bien en su origen la agenda de lucha feminista por la igualdad es ya de sí global, internacional y con vocación de solidaridad universal, en las últimas tres décadas el trabajo por interpretar los derechos de las mujeres como derechos humanos (Okin 1999, 2000; Jaggar 
2000) ha materializado esa vocación tanto en un anclaje legal de indudable fuerza, como en una nueva concienciación de lo que se quiere expresar por “igualdad de género". La década de las mujeres de Naciones Unidas (1976-85), así como el nuevo escenario de entendimiento global de los derechos humanos después de la guerra fría, son parte inequívoca de este ímpetu. El feminismo ha mostrado, desde los 90, un interés en comprender la situación de las mujeres en el mundo de manera coordinada, creando foros de diálogo transnacional que pudieran recoger la multiplicidad de experiencias de las mujeres. En este diálogo el feminismo ha tratado de superar un discurso de derechos de las mujeres muy centrado en la experiencia de una cierta clase de mujeres: occidentales, blancas, de clase media y heterosexuales. Como consecuencia y reflejo de ese diálogo empezará a usarse cada vez con más fuerza la etiqueta plural de "feminismos". La concienciación de una ciudadanía global (Kaldor 2003), junto con esta visión de la abrumadora variabilidad humana, han guiado un debate dentro del feminismo acerca de cómo entender ese sujeto mujer para el que estamos reclamando derechos humanos. Este debate, no sólo es importante a efectos teóricos y académicos, sino que tiene repercusiones en la vida de las mujeres. Cómo interpretemos la identidad de sexo/género tiene consecuencias importantes a la hora de reclamar derechos, enunciarnos como sujetos en esos derechos y, en definitiva, luchar por mejorar las vidas de las personas afectadas por muchas de las situaciones de detrimento que vivimos en el mundo actual.

Reinventar esa vocación cosmopolita, después de derrumbado el exclusivo "feminismo blanco", supondrá partir de una tensión interna al mismo feminismo, y que es una de importante calado. Se trata, ni más ni menos, de averiguar cómo entienden el sujeto los feminismos. Sin entrar en profundidad en este debate, pues no es el tema de este escrito, es necesario apuntar que hay dos polos en tensión:

a- El polo de apostar por conformar ese sujeto "mujer" que pueda servir de referente universal para esa lucha por la igualdad, evitando el peligro de caer de nuevo en un "feminismo exclusivo y excluyente" y occidentalizado (Mohanty 1984). Es decir, este es el polo que permite invocar un sujeto político universal, "las mujeres".

b- El polo de resistir a cualquier representación universalista por contradecir el mismo espíritu de los movimientos de mujeres, quienes en sus luchas visibilizan experiencias muy diferentes y difícilmente unificadas en la enunciación de un sujeto único. Es decir, este es el polo que impide invocar un sujeto político universal, "las mujeres".

El reto del feminismo cosmopolita que aquí presentamos será poder articularse como teoría política respondiendo a la vez a esos dos elementos en tensión. Por ello, no podrá perderse ni en un falso universalismo saturado de 
un sujeto occidental que no deja espacio a las diferencias; ni en un abandono del universalismo por quimérico, con la proclama de que es imposible hablar de un concepto de sujeto colectivo. Habrá de ser un cosmopolitismo que pueda hacer servir un sujeto "mujeres" sin caer en ningún esencialismo, ni negar diferencias y diversidades, habrá de ser a la manera como la identidad interseccional propone (Crenshaw 1989) cuando nos explica que la identidad está en la intersección de diferentes relaciones de poder. El reto es componer un sujeto que pueda hacer frente a las amenazas constantes de un neoliberalismo global especialmente desempoderante con los débiles, entre ellos la mayoría de las mujeres, a quienes la globalización neoliberal constantemente precariza (Butler 2015).

En este sentido, y en nuestra propuesta, ese sujeto ya no es "mujer", ni se identifica con ninguna esencia, sino que es un sujeto, si bien inestable, claramente identificable en su desempoderamiento continuo por estructuras, que aun usando el discurso de la igualdad, cometen traiciones continuas a la misma. Esta es la llamada "precarización de la vida" que se da cada vez más desde un neoliberalismo globalizado que se estructura en jerarquías explotadoras, ocultas muchas veces en el lenguaje de la democracia global y los derechos humanos (Chant 2008). Por ello, y como algunas autoras señalan (Reilly 2007; Mohanty 1984, 2003), será importante para la tarea de lucha por la igualdad, alcanzar un feminismo cosmopolita que utilice los derechos humanos para retar las desigualdades socioeconómicas.

El desmembramiento de un sujeto colectivo "mujer" (lo que hemos llamado el segundo polo) ha supuesto en las últimas décadas la proliferación de discursos que, aun teniendo vocación feminista, no llegan a explicar las cuestiones de explotación y opresión sistemática que las mujeres como colectivo sufren en diferentes lugares y tiempos. En ese sentido, las narrativas neoliberales tienen legitimidad para rechazar la importancia de las experiencias colectivas, situándose así, cómodamente, en un discurso que sólo valora la experiencia individual, despojándola de cualquier posibilidad de ser verdaderamente política. La experiencia de opresión sin posibilidad de ser social, quedándose meramente en narrativa individual, se colapsa así en su oportunidad de convertirse en crítica y transformadora. Ello puede ser aprovechado rápidamente por un neoliberalismo que tiene muy fácil la desarticulación de cualquier experiencia al convertirla en una mera mercancía. Y ello vuelve banal e irrelevante las críticas feministas hechas sin el recurso a un sujeto político colectivo (Stern 2016). El eslogan de Kate Millet de los setenta de "lo personal es político", tan importante para la lucha feminista, se desvanece en cuanto pierde las posibilidades de convertir la experiencia personal en una sintonía de experiencias personales en diálogo, unificadas bajo un sujeto común en cuyo recurso y nombre se hacen políticas. El eslogan 
entonces se vuelve "lo personal es meramente personal", privatizando y despolitizando así la experiencia. Como Mohanty (2013: 972) de manera clara afirma:

Here, political agency itself is redefined as an act of consumption, and I would argue that theory -feminist and/or antiracist- is trafficked as a commodity disconnected from its activist moorings and social justice commitments.

La crítica feminista corre el riesgo de convertirse así, ella misma, en una mercancía asequible para el consumo neoliberal. Tornada en una teoría política discursiva, meramente representacional, desconectada de lo colectivo, lo social y lo material y sin posible conexión con las relaciones de poder subyacentes a toda experiencia, es fácilmente asimilada en los discursos y propuestas políticas de las instituciones defensoras del proyecto neoliberal; podemos decir que es incluso cooptada por esas instituciones (Calkin 2015). Así, la estrategia de cooptación ha sido observada por las feministas (Hunt 2002, Kantola \& Squires 2012, Mohanty 2013), quienes alertan del peligro de las políticas pro-mujeres (por ejemplo las políticas denominadas Business-woman) que acaban vaciando de contenido las tesis de transformación de las sociedades. Es entonces cuando el discurso de "justicia-de-género" se hace compatible con formas de prácticas económicas y de desarrollo contaminadas por el neoliberalismo más aberrante y deshumanizador ${ }^{2}$. El informe del Banco Mundial publicado en 2011 (The World Development Report 2012 on Gender Equality and Development) es buena prueba de ello, como veremos más adelante.

Las que habíamos definido más arriba como fuerzas contrapuestas o polos en tensión (la afirmación de un sujeto universal o la disolución, por etnocéntrico y falso, del mismo) pueden concurrir en la situación de favorecer ambos un discurso neoliberal que, legitimado por unos feminismos (universalistas) u otros (poscoloniales), campa a sus anchas en las instituciones democráticas. Estas utilizan convenientemente la etiqueta "igualdad de género" o "diferencias de las mujeres", para cumplir con un lenguaje políticamente correcto que habla de "empoderamiento de las mujeres" (Eyben \& Napier-Moore 2009) a la vez que impulsan realidades desiguales, injustas y desempoderantes.

Nuestra propuesta es que el cosmopolitismo ha de impedir tanto un universalismo vacío y ciego a las diferencias, como tan lleno de ellas que en realidad no activa ninguna lucha colectiva, ni vertebra reacciones a las opresiones vinculadas a mecanismos de poder. Las diferencias en las experiencias de las mujeres existen; pero, los mecanismos de poder, cada vez más globalizados,

${ }^{2}$ Hozic \& True (2016) nos ponen el ejemplo de los microcréditos. Estos, si son diseñados e intencionalmente vendidos como inversión, y no para cubrir necesidades básicas, pueden acabar dejando a las mujeres en la desposesión y la deuda, en vez de empoderarlas para ser dueñas de sí misma y su familia.

Araucaria. Revista Iberoamericana de Filosofía, Politica y Humanidades, año 19, no 37. Primer semestre de 2017. Pp. 301-325. ISSN 1575-6823 e-ISSN 2340-2199 doi: 10.12795/araucaria.2017.i37.15 
que oprimen a las mujeres también. Un cosmopolitismo feminista (y un feminismo cosmopolita), o igualitario, debe poder articular precisamente en qué medida la justicia y la igualdad globales son posibles, sin deslegitimar las diferencias, pero sin perderse y diluyendo la agencia política en ellas. Por decirlo brevemente, el cosmopolitismo feminista por el que abogamos ha de articular un sujeto político que siendo universal ("las mujeres") incorpore las diferencias de las mujeres en las relaciones de poder.

Veamos antes las amenazas para el avance de la igualdad de género como argumentos para la necesidad de articular un cosmopolitismo feminista.

\section{Igualdad de género y "Smart Economics". El caso del Banco Mundial}

Si bien la globalización en forma de interpretación de los derechos de las mujeres como derechos humanos ha conllevado mejoras para la igualdad de las mujeres, básicamente a través de medidas legales y la aplicación de la estrategia de mainstreaming ${ }^{3}$ (Caglar, Prügl \& Zwingel 2016), sigue siendo urgente mantener una mirada crítica que visibilice en nombre de quién y para quién se realizan esos cambios.

La vigilancia global que ejercen las agencias internacionales sobre los estados en materia de igualdad de género ha ido in crescendo. De hecho, la presión ejercida en materia de igualdad por los organismos internacionales puede haber llevado al sector privado y a las grandes corporaciones a comprometerse con la agenda de igualdad, jugando así un mayor papel en la gobernanza de género y el desarrollo de programas igualitarios (Bexell 2012). Sin embargo, un análisis crítico (Elias 2013, Prügl 2017) nos indica cómo el trabajo en igualdad de género de organizaciones como el Foro Económico Mundial (WEF) o el Banco Mundial (BM) son totalmente compatibles con políticas y prácticas neoliberales que promocionan y priman formas de crecimiento económico de acuerdo a las leyes e intereses del mercado. Esto puede poner de manifiesto la maleabilidad de las agendas feministas manejadas por la visión neoliberal; en el sentido de que el discurso de igualdad de género suele estar en gran parte despolitizado y alejado de perspectivas críticas feministas. De hecho, y como suele ser denunciado por algunas voces críticas (Mohanty 2013), el marco de análisis de las desigualdades de género y de las propuestas para corregirlas

\footnotetext{
${ }^{3}$ Para intentar remediar el déficit en logros igualitarios el Tratado de Amsterdam de 1997 de la Unión Europea institucionalizó la estrategia conocida como "mainstreaming de género". Según este planteamiento se trata de incorporar la perspectiva de género en todas las áreas de la política y a todos los niveles para asegurar el camino a la igualdad entre hombres y mujeres. El objetivo final es la incorporación del análisis de género en la elaboración y diseño de cualquier política comunitaria en cualquiera de los campos a legislar. Como han señalado algunas voces (Jacquot, 2003) estas políticas de mainstreaming ponen de relieve el hecho de que con las tradicionales políticas de igualdad de oportunidades no se ha alcanzado el nivel esperado de igualdad entre hombres y mujeres.
}

Araucaria. Revista Iberoamericana de Filosofía, Politica y Humanidades, año 19, nº 37. Primer semestre de 2017. Pp. 301-325. ISSN 1575-6823 e-ISSN 2340-2199 doi: 10.12795/araucaria.2017.i37.15 
desde las instituciones y corporaciones internacionales se basan en una comprensión del género absolutamente esencialista (Elias 2013: 154); algo que es considerado por todos los feminismos como el sustento principal del patriarcado y sus desigualdades.

Para entender este "matrimonio de conveniencia" entre la agenda de igualdad y las instituciones internacionales creemos relevante el análisis del informe del Banco Mundial (The World Development Report 2012 on Gender Equality and Development). No sólo por la importancia y alcance de la organización que lo propone, sino por las implicaciones prácticas que dicha organización tiene sobre las vidas de millones de personas. En este sentido, es importante recordar que el Banco Mundial es la principal institución mundial en diseñar, proponer y llevar a cabo políticas de desarrollo.

Formulado como un informe que sigue la estela de la estrategia de la Cuarta Conferencia Mundial de las Naciones Unidas en Pekín sobre las Mujeres (en 1995), el informe de 2012 del Banco Mundial enfatiza la necesidad de invertir en mujeres y niñas para poder tener mejores resultados en el desarrollo de pueblos, comunidades y generaciones futuras, e incluso en representación democrática en las instituciones:

Una mayor igualdad de género puede incrementar la productividad, mejorar los resultados en materia de desarrollo para la próxima generación y hacer que las instituciones sean más representativas (BM 2011: 1).

Como algunas críticas señalan (Razavi 2012; Chant 2012) el Banco Mundial se afana en acentuar en sus discursos los beneficios de la globalización para las mujeres. Sin embargo, el término "globalización" es reducido a una visión saneada de liberalización de los mercados y a una aplicación generalizada de las tecnologías de la información y la comunicación; dejando así fuera de su interés aspectos controvertidos de la globalización, como pueden ser la liberalización financiera, las metas de la inflación y las profundas reformas que se están llevando a cabo en el sector público para aminorarlo. La crisis de la última década ha dejado claro en sus efectos que la economía, tanto a nivel macro como micro, está atravesada por cuestiones de género (Karamessini 2014, Prügl \& True 2014, Hozic \& True 2016). Sin embargo, los análisis de las organizaciones internacionales, como el Banco Mundial o el Foro Económico Mundial, dedican poca reflexión a indagar cuál es la estructura de las relaciones de género que convertidas en relaciones de poder sustenta cualquier propuesta económica, incluso las bien intencionadas; es decir, las que incluyen entre sus objetivos la igualdad de género. Uno de los mejores ejemplos de esto es la propuesta de "Smart Economics" realizada por el Banco Mundial. 
El informe del Banco Mundial de 2012 (aunque publicado en 2011) menciona el término "Smart Economics" como una propuesta vinculada a la igualdad de género. En palabras del propio presidente de dicha organización, Robert B. Zoellick, en la presentación del informe (World Development Report 2012: xiv):

Gender equality is at the heart of development. It's the right development objective, and it's smart economic policy. The World Development Report 2012 can help both countries and international partners think through and integrate a focus on gender equality into development policy making and programming.

Aunque el término "Smart Economics" sólo vuelve a aparecer en la contraportada de este informe, fue un término suficientemente impactante en un informe anterior: Gender Equality as Smart Economics: World Bank Group Gender Action Plan. First Year Progress Report (2007-2008). En este se habla de la "renovada estrategia" de la organización al proponer el empoderamiento económico de las mujeres y "la igualdad de género como economía inteligente" ("gender equality as Smart Economics") en el centro del discurso del desarrollo. Se define como una "estrategia rentable". De hecho, la traducción al castellano de tal informe traduce "Smart Economics" como "estrategia rentable" y en el mismo título como "estrategia económica acertada". En la traducción al castellano aún queda más claro el principal objetivo del Banco Mundial en sus estrategias en la última década respecto a la igualdad de género y desarrollo. Convertido casi en un "mantra" el término "Smart Economics" ha sido empleado posteriormente en informes de otras organizaciones como Care, UNICEF, UN_ HABITAT o incluso la Fundación de la compañía NIKE al lanzar su campaña en 2008 "Girl Effect" (Chant 2012). La dialéctica subyacente a la estrategia de la "Smart Economics" es la de la posibilidad de un retorno fácil en términos de desarrollo si se invierte en las mujeres, especialmente si se hace en la etapa de la adolescencia y juventud. Como muchas críticas feministas recalcan (Benería 2102, Calkin 2015, Chant 2012, Zuckerman 2007, Zuckerman \& Qing 2003, Prügl 2017) la idea parece ser la de invertir en mujeres jóvenes para promover el desarrollo y beneficiar a otros y a la sociedad en su conjunto, dejando claro que el objetivo principal es "hacer que las mujeres trabajen para los mercados" (Chant 2012: 202). Esta instrumentalización del género hace de las mujeres un "conducto político" (Molyneux 2006, 2007) al servicio de otros, especialmente la familia. Ello conlleva una esencialización del concepto de mujer, que sigue entendiéndose dentro del paradigma patriarcal heteronormativo como un sujeto femenino y heterosexual, a cargo de la familia y especialmente de hijos e hijas. Como Zuckerman (2007) señala, esta estrategia sigue ignorando el imperativo moral de alcanzar los derechos de las mujeres como plenos derechos en igualdad con los hombres. Si tomamos en cuenta los Objetivos del 
Desarrollo del Milenio que la ONU elaboró en el año 2000 para ser cumplidos en el 2015, nos damos cuenta de que el "empoderamiento económico de las mujeres" se toma como "solución” a la pobreza global; remarcando así la idea de la "Smart Economics" en la consideración de la igualdad de género y el empoderamiento de las mujeres en términos de ganancia para la agenda política y económica neoliberal, caracterizada por el fundamentalismo de los mercados, la desregulación y el desarrollo liderado por las corporaciones (Calkin 2015). Como Larner (2000) nos dice, el neoliberalismo es multifacético y se apoya básicamente en tres dimensiones: un conjunto de políticas económicas; un viraje desde los estados a los mercados, abandonando las políticas de bienestar; y, la privatización de los servicios públicos. La lógica del mercado termina así invadiendo todos los aspectos sociales y políticos de la vida, en una flagrante negación del mismo sentido de lo social y político. El problema que está a la base es el de que estas instituciones globales, con el Banco Mundial a la cabeza, entienden que el crecimiento económico por sí solo puede romper las barreras de la desigualdad entre los géneros (Benería 2012). Sin embargo, y desde una abrumadora evidencia al respecto, ni las normas culturales, ni las normas opresivas, ni la violencia contra las mujeres, se terminan con el crecimiento económico (Waylen 2008). De hecho, y como Kabeer (2016) recientemente nos señala, podemos encontrar evidencia de que la igualdad de género contribuye positivamente al crecimiento económico, pero no hay evidencia de la relación reversa. Esta asimetría revela que si bien la igualdad de género contribuye al crecimiento $^{4}$, no hay consistencia probada de que este beneficie de alguna manera a las mujeres.

La introducción en las instituciones globales mencionadas de ciertos aspectos de la lucha por la igualdad de las mujeres, así como el uso extensivo del lenguaje de los derechos de las mujeres como derechos humanos (Eisenstein 1996) y de la acentuación de las ventajas que para el desarrollo económico y la democracia tiene la igualdad de género, está demostrando una cooptación del feminismo por parte del neoliberalismo y sus representantes (Calkin 2015). Esta cooptación, a veces consentida por las feministas, niega la posibilidad de reformular el sujeto mujeres en la diversidad y la disensión que el movimiento y sus estrategias de empoderamiento e igualdad necesitan. Con la institucionalización neoliberal de la igualdad de género, si bien se consigue una indudable visibilidad de algunos problemas graves para las mujeres, como el de la violencia en sus diferentes formas, se opaca el hecho de que son las

\footnotetext{
${ }^{4}$ Por señalar algunos aspectos de la contribución al desarrollo y al crecimiento que la agenda de igualdad de género puede tener podemos mencionar dos (Kabeer, 2016): (1) Dadas las responsabilidades reproductivas las mujeres suelen invertir sus ganancias y recursos en la familia, sirviendo así para incrementar la productividad de la siguiente generación. (2) La igualdad de género ayuda a distribuir las habilidades innatas que podamos tener, tanto hombres como mujeres. Esto ayuda a distribuir los recursos humanos y ello se traduce, a su vez, en mejora de la productividad.
} 
políticas económicas neoliberales las que imposibilitan un cambio sustancial que transforme las sociedades en iguales y, por tanto, más justas. Como hemos visto, la idea de un desarrollo basado en las iniciativas de la "Smart Economics", velará, en definitiva, por un desarrollo centrado en la visión de las mujeres y las niñas como proveedoras de cuidado para sus familias. El empoderamiento de las mujeres no pasa, en este sentido, a entenderse como personal y político y que puede ir deshaciendo prejuicios patriarcales, sino como eficaz para "generar riqueza". Por eso mismo, los informes internacionales de las principales agencias, como la ONU o el Banco Mundial, en temas de igualdad de género no integran de manera seria la reflexión sobre el gran volumen de trabajo no pagado que realizan las mujeres en el mundo (Chant 2012). La identidad de las mujeres, en sus roles de cuidadoras, madres, esposas e hijas, queda así convenientemente inmovilizada en el discurso de igualdad de géneros de las organizaciones internacionales. De alguna manera, las estructuras patriarcales centradas en la asignación universal de las responsabilidades primarias por el trabajo de cuidado de la familia a las mujeres no cambia la calidad de vida de las mujeres, aunque sí puede tener efectos positivos para la productividad de las generaciones futuras de trabajadores y trabajadoras (Kabeer 2016: 315).

Esta orientación inmovilista en el nivel de gobernación global de las instituciones internacionales se ve también reflejada en el nivel estatal, que comparte con las anteriores instituciones la compatibilidad del discurso de la igualdad de género con las prácticas neoliberales contrapuestas a esa igualdad. La reconstrucción del papel de sujetos de las mujeres sugiere, así, que las políticas públicas funcionan como sistemas de legitimación de sentido, que, como dice Nancy Fraser (1989: 146) "construyen a las mujeres y sus necesidades según ciertas específicas $-\mathrm{y}$ en principio, cuestionables o disputables- interpretaciones". Y si la identidad de las mujeres acaba siendo resubordinada en este sentido, más lo será la de aquellas mujeres objeto de los programas de desarrollo que acabamos de analizar. Estas mujeres pueden acabar siendo vistas como una ciudadanía aparte, marcada por género, clase, raza y sexualidad. En este sentido, Fraser (1989) nos alerta de cómo el "aparato burocrático del estado de bienestar acaba posicionando a las mujeres pobres que reciben asistencia social como ciudadanas de segunda categoría, o peor, como "clientes dependientes"".

El trabajo de algunas feministas (Álvarez 1998) sugiere que el imperativo de "incorporar el género" a la planificación para el desarrollo puede estar llevando a los Estados y a las organizaciones inter-gubernamentales a "consultar" a las ONGs feministas locales, nacionales y transnacionales más en su capacidad técnica en género, que en su capacidad como organizaciones de la sociedad civil o del movimiento feminista que promueven la ciudadanía plena para las mujeres. Es decir, más como técnicas y menos como ciudadanas. Los 
grupos de "expertas en género" se han convertido para muchas instituciones públicas en los interlocutores cualificados, reemplazando así a los grupos de mujeres feministas, apartando con ello, sutil y veladamente, a los movimientos de la sociedad civil.

Con esta tendencia de los estados modernos y lo que Álvarez (1998) llama “el 'boom' en la sub-contratación de ONGs" se está favoreciendo cada vez más a los sectores más tecnocráticos de la sociedad civil, dejando a un lado las cuestiones políticas y de principios que fundamentan el movimiento y la agenda feminista.

Este tipo de discurso tecnocrático está completamente en consonancia con un progresivo desmantelamiento del Estado y de la esfera de la política social, incrementado ahora por la crisis financiera con una redefinición neoliberal de las funciones del Estado, que amenaza con desarticular el estado del bienestar como protección de la ciudadanía para pasar a ser responsable último ante los desmanes de los actores principales del neoliberalismo. Los mercados y la iniciativa privada han sido considerados como los mecanismos más eficientes para lograr el crecimiento económico y para proporcionar la mayoría de los servicios a la mayoría de la gente, y se han convertido en los mecanismos preferidos y casi exclusivos para la provisión de servicios, sustituyendo, al Estado; quien facilita y promociona esta sustitución (Álvarez 1998).

De esta manera los estados modernos, junto a instituciones internacionales como el Banco Mundial, han reinventado el patriarcado recodificando las relaciones de género y atendiendo normalmente sólo a lo que se considera "situaciones extremas" o "patologías sociales" (como la violencia contra las mujeres que acaba siendo vista como una condición patológica o un problema psicológico individual, en vez de una expresión o consecuencia "normal" de la subordinación de la mujer), dejando de lado la verdadera lucha por la igualdad, secuestrada por los intereses neoliberales del estado y de las agencias globales.

Los procesos globales, en sintonía con los estados, promueven así, un discurso igualitarista sin una verdadera transformación de las jerarquías patriarcales; manteniendo con ello la desigualdad. A su vez, el sujeto de estos discursos igualitaristas está despolitizado, en cuanto que es un universal "mujeres" esencializado en sus cualidades meramente cuidadoras, y desmembrado en sus múltiples experiencias personales. Por ello la globalización interpretada simplemente en lo económico atenta contra un verdadero concepto de cosmopolitismo político y ético, como las políticas de la "Smart Economics" demuestran. Esa falta de cosmopolitismo amenaza las posibilidades que una globalización puede suponer para la igualdad. La visión neoliberal de la globalización conlleva, así, un reforzamiento de las estructuras patriarcales, a la vez que cínicamente mantiene el discurso de "igualdad de género" y atención a la diversidad y a las diferencias. 
Se tratará, por ello, de proponer un concepto de globalización y de gobernanza global que recoja tanto un cosmopolitismo transformador $\mathrm{y}$ crítico como una verdadera igualdad entre las identidades de sexo/género. Un cosmopolitismo de este cariz no se puede conseguir si antes no hemos sacudido el concepto mismo de cosmopolitismo de ciertos legados patriarcales que han generizado el mismo concepto de individuo cosmopolita y de sociedad global. Para ello será necesario relacionar ese concepto de cosmopolitismo, tanto con la ética y la economía del cuidado (Tronto 2013), como con las propuestas desde la interseccionalidad (Wilson 2015).

Veamos ahora cómo habría de ser ese nuevo cosmopolitismo.

\section{Cosmopolitismo feminista}

Por todo lo dicho, lo que en este escrito proponemos es la formulación y activación de un cosmopolitismo feminista como mecanismo para irrumpir en el "fácil tránsito del feminismo al neoliberalismo" (Orloff \& Shiff 2016), que parece haber ocurrido en las instituciones y organizaciones internacionales, las cuales cuentan con poder para interpretar qué son las mujeres. Si no podemos constituir un feminismo que rompa la fácil alianza que el neoliberalismo y sus instituciones han tramado corremos el riesgo de neutralizar las posibilidades de transformación social que las agendas feministas originalmente conllevan.

Pensamos que un feminismo capaz de activar transformaciones sociales relevantes ha de poder desactivar, a la vez, los efectos negativos que la globalización está imponiendo, tanto a personas - mujeres y hombres- como al planeta mismo. Estudios recientes señalan (Kabeer 2016) que un cambio de actitudes en la consideración de la igualdad de género y las políticas para promoverlas no cambia automáticamente las prácticas patriarcales; sino, que hace falta una acción colectiva de las mujeres basada en organizaciones autónomas y en alianza con otros grupos. Si tenemos en cuenta los flujos de migración actuales, los cuales recrean nuevas condiciones de desigualdad de género, se hace evidente que ese cambio de actitudes y políticas es necesario a escala global. Más si entendemos que la migración internacional visibiliza la creciente mercantilización del trabajo de cuidado de las mujeres a escala global (Benería, Deere \& Kabeer 2012). Por ello, es importante la habilidad de las mujeres para movilizarse colectiva y globalmente lo que puede tener más peso a la hora de lograr transformaciones significativas respecto al progreso de la igualdad de género (Kabeer 2016: 217). Ello implica la constitución y estabilización de un sujeto mujeres que pueda ser universal y que accione esas movilizaciones colectivas, que ahora hemos visto que han de tener como objetivo transformaciones globales. Lo que consideramos en este escrito es 
que para ello será necesario recuperar un sujeto cosmopolita fuertemente comprometido con la igualdad de género, y que por supuesto vaya más allá del "cosmopolitismo banal" fácilmente mantenido por algunas instituciones y corporaciones, como Beck (2006) denuncia.

Cómo habrá de ser ese cosmopolitismo es parte del debate que aquí proponemos y que consideramos que es clave para superar las divisiones y enfrentamientos de los feminismos en las últimas décadas, y que hemos explicado más arriba como dos polos en tensión dentro del feminismo. El objetivo principal habrá de ser fortalecer la lucha por la igualdad frente a las crecientes amenazas de una globalización neoliberal que atenta contra cualquier visión cosmopolita comprometida con la igualdad y la justicia. La teoría feminista mantiene una actitud de sospecha frente a cualquier universal, aunque a la vez necesita proclamarlo en su vocación de lucha por la igualdad y la justicia. Gran parte de la investigación realizada en el área de estudios de género se ha centrado en las últimas décadas precisamente en encontrar la forma de poder combinar ambos: un rechazo al sujeto universal (por falso y patriarcal); y una querencia por intentar reformular un sujeto que pueda articular las demandas de igualdad desde la condición de dignidad del ser humano (y por ende, desde la misma base ideológica de los derechos humanos). Pensamos que este difícil trasiego entre la crítica y la aceptación de lo universal constituye un escenario que posibilita la reconsideración del cosmopolitismo como sustrato teórico que nos permita conformar ese sujeto.

\subsection{El concepto de cosmopolitismo crítico}

El término cosmopolitismo se ha estudiado normalmente en teoría política asociado a la tradición de Leibniz, Wolf y Kant y relacionado con los conceptos de universalismo global, ciudadanía global y república mundial. Como nos cuenta Grande (2006) el concepto de cosmopolitismo fue recuperado en los noventa, para ser articulado en relación al proceso de globalización económica, como un concepto normativo que pudiera articular prácticas políticas para contestar a los efectos negativos de ese proceso. El cosmopolitismo puede verse así en relación tensa con la globalización (Pieri 2014), e incluso como una crítica fundamental y definitiva a los procesos de globalización que están aconteciendo hoy en día (Delanty 2012a).

La actual globalización neoliberal y las iniciativas llevadas a cabo desde los organismos internacionales más influyentes se aplican como nuevas formas de gobernanza que en realidad son expresiones de intereses privados de grandes corporaciones (Prügl \& True 2014). El aspecto normativo del término cosmopolitismo impone un ejercicio de revisión (incluso de oposición) a las condiciones prevalentes de vida, señalando alternativas al status quo. Por ello 
Delanty (2012b) nos advierte que esta característica de buscar alternativas se confunde normalmente con una visión puramente idealista, sin ningún arraigo en la realidad; considerado así un proyecto, que si bien puede comportar a largo plazo cambios importantes a nivel socio-económico y político, es "demasiado idealista". Sin embargo, y en propuesta de Delanty (2012a), el cosmopolitismo habría de entenderse no sólo en su sentido normativo, sino también crítico con las realidades actuales. Es más, sin alguna noción de sociedad alternativa el elemento normativo del cosmopolitismo no tiene sentido (Delanty 2006).

El idealismo del que es acusado el cosmopolitismo se desvanece si lo entendemos en su vocación de transformación de la realidad buscando alternativas críticas y reales a los graves desajustes que provoca la globalización. Por ello, la propuesta de Delanty (2012a: 41) de un "cosmopolitismo crítico" nos parece útil para nuestro propósito:

The idea of a critical cosmopolitanism is relevant to the renewal of critical social theory in its traditional concern with the critique of social reality and the search for immanent transcendence. It also offers a route out of the critique of domination and a general notion of emancipation that has so far constrained critical theory. It provides a promising approach to connect normative critique with empirically based analysis focused on exploring new ways of seeing the world. Such forms of world disclosure have become an unavoidable part of social reality today in terms of people's experiences, identities, solidarities and values.

A la vez, este cosmopolitismo escapa de la crítica contraria, que le sitúa en alianza con el capitalismo de la era global con dinámicas de libre mercado y flujo de capitales. En este sentido un cosmopolitismo meramente entendido en su aspecto de ley universal para el libre movimiento de personas puede funcionar en realidad como excusa para la economía capitalista de mercado, pudiendo convertirse entonces en una ideología de la explotación capitalista (Sorensen 2016). Esta manera de enfocar el concepto de cosmopolitismo obviamente no nos sirve para sustentar un sujeto político capaz de transformar la realidad material.

La propuesta de Delanty (2012a, 2012b) parte de la tradición de la Teoría Crítica de la Escuela de Frankfurt a lo largo de sus tres generaciones. Sin embargo, y como el propio autor nos revela (Delanty 2012a), su propuesta despoja al término de toda pertenencia europea y occidental. Si bien la genealogía y la historia del concepto remiten a una tradición europea, el nuevo significado se vincula a un momento post-occidental, en el que lo importante ya no es lo local y lo universal; sino la interfaz de lo local y lo global que se hace realidad en las experiencias concretas. Entendido así, como una condición de "apertura al mundo", comporta transformaciones del individuo y de la sociedad en el encuentro con el Otro/Otra. El cosmopolitismo comprendido de esta forma 
se distancia de la globalización y del internacionalismo y se convierte en una crítica a ambos. El carácter dialógico de este cosmopolitismo, ya apuntado por los autores de la Teoría Crítica, especialmente Habermas (2003), conlleva una apertura hacia nuevos horizontes de sentido y de compromiso con el Otro/Otra. Es este compromiso el que, a su vez, aleja a este cosmopolitismo del peligro de ser un aliado de la explotación capitalista.

En este sentido, el concepto de cosmopolitismo que una justicia social que profundiza en la plena igualdad de género requiere es uno vinculado a una lógica material de producción y distribución que sea distinta a la actual lógica material de los mercados del capitalismo global. Y por ello, ha de ser un cosmopolitismo que no sólo refiera a la ética y la moralidad, sino también, y necesariamente, a la política y a la ley (Sorensen 2016), y a la economía y a la distribución (Brock 2013). Habrá de ir más allá de un programa liberal que tiene como objetivos la paz y la justica globales basándose simplemente en la moral individual y los derechos humanos. Este cosmopolitismo liberal no sirve a la causa de la igualdad de género (ni de otro tipo). Y de hecho nos muestra las tensiones, apuntadas por ejemplo por Benhabib (2006), entre liberalismo y democracia, que son a su vez entre cosmopolitismo y democracia (Sorensen 2016). Un cosmopolitismo exige por ello, al menos, tres ámbitos de reflexión y praxis: la moral, la legal-institucional y la política-social (Marchetti 2012). Un cosmopolitismo comprometido con la transformación igualitaria de la realidad habrá de activar armónicamente esos tres ámbitos. Así, si el cosmopolitismo refiere a la ética (Appiah 2006), esta a su vez, está indefectiblemente unida tanto a las estructuras legales como a la distribución. Pensemos que todo cosmopolitismo tiene como componente central el reconocimiento de la misma valía moral de todos los individuos y los mismos derechos a igual respeto, y que ambos requisitos atañen tanto a lo formal como a lo material (Brock 2013).

Si bien es cierto que para un cosmopolitismo eficaz en este sentido será necesario mantener la estructura epistemológica del universalismo, podemos reformular este último concepto para sacudirlo de cualquier apropiación que pueda anular las diferencias. Así, y como nos propone Delanty (2012a) podemos entender el universalismo de forma que no exija un asentimiento universal o que todo el mundo se identifique con una interpretación única: "Depending on the social context or historical situation social actors will interpret universal rules differently and put them to different uses" (Delanty 2012a: 42). Esta forma de cosmopolitismo presupone, así, un universalismo "débil", que pueda ser compatible con el relativismo, en el sentido como Sahlins (2000: 21) lo formula: "the provisional suspension of one own judgments in order to situate the practices at issue in the historical and cultural order that made them possible". 
De alguna manera ese llamado "universalismo débil" propone la contestación del concepto histórico de universalismo tanto como su recuperación renovada. Se trata de reivindicar el concepto en una forma nueva y libre de las connotaciones coloniales y patriarcales para que pueda servir a la causa de la igualdad, y en nuestro caso, a un cosmopolitismo feminista.

\subsection{El sujeto (igualitario) del cosmopolitismo}

¿Qué sujeto es el de este cosmopolitismo que estamos intentando fraguar para que sirva a la agenda de igualdad de género?, ¿Podrá ese sujeto volver fructífera la tensión de los dos polos en el feminismo que hemos comentado al principio de este escrito? Si bien la recuperación universalista de ese sujeto es condición sine qua non para el cosmopolitismo, será necesario mantener lo que Mignolo (2000) llama "locus material de enunciación". En este sentido, la fuerza de lo local, si bien en tensión con lo universal (Mendieta 2009), es parte de la interlocución a la hora de reconocer qué entendemos por humanidad y poder forjar así ese sujeto universal. La estructura institucional moral, política y legal de una propuesta efectiva de cosmopolitismo ha de permitir, a la vez, la cohabitación en comunidad global y la diferenciación en la humanidad. Por eso el cosmopolitismo ha de ser un juego dialéctico constante entre singularidad y universalidad, localización y desplazamiento, enraizamiento y desenrraizamiento, asentamiento y movilidad. En esto hay cierta corrección a la definición cosmopolita kantiana, la cual, y en observación de Nussbaum (1997) no llega a desprenderse de la perspectiva de la cultura europea, cayendo, por ello en asunciones eurocéntricas, racistas y sexistas (Mendieta 2009). El peligro de no encontrar esa comunidad global, o seguir enunciándola sin efectivamente articularla, es la de seguir haciendo fuerte un sujeto y una humanidad aptas y útiles para un mundo que parece estar basado en el fetichismo de la mercancía, la explotación de trabajadores/as y el capitalismo neoliberal (Nassem \& Hyslop-Margison 2006: 59). Y esta realidad, como hemos visto más arriba, no sólo juega en contra de la igualdad, sino que la imposibilita.

Un concepto estrecho, y falsamente universal, de derechos humanos o un sujeto de esos derechos fundamentado en un universal de corte ilustrado, puede mantener la desprotección de las víctimas del neoliberalismo. Por ello un feminismo cosmopolita, o un cosmopolitismo igualitario, está comprometido con una interpretación crítica de los derechos humanos y el concepto de universalidad, como Reilly (2007) afirma en su artículo "Cosmopolitan Feminism and Human Rights". Esta autora propone un cosmopolitismo feminista que no esté basado en la asunción de que las mujeres están unidas por una identidad de género común o una experiencia de opresión patriarcal 
a través de diferentes regiones y culturas. En vez de eso Reilly propone un feminismo cosmopolita como una estructura de proceso abierto orientado al diálogo dentro y a través de los movimientos de mujeres más allá de líneas divisorias identitarias. Utilizando el concepto de interseccionalidad comentado más arriba, Reilly (2007) parte de la línea de trabajo de que las mujeres no son un grupo monolítico con una agenda común y "natural". La concepción antiesencialista llevada a cabo por gran parte de la teoría feminista de la segunda ola promueve un reconocimiento de la experiencia de las mujeres que se mueve en variables múltiples de identidad, siendo el género sólo una de esas variables. Pensamos que la propuesta de un cosmopolitismo feminista tiene que estar atento a esta multiplicidad identitaria a la hora de entender cómo ha de darse un diálogo que pueda negociar los principios universales que puedan regir el cosmopolitismo. En este sentido, el sujeto universal cásico del que parte la ética del discurso (Held 2010) y que se sitúa en el "razonamiento imparcial" como base del "auténtico diálogo" no tiene en cuenta la realidad de los sujetos que ocupan posiciones marginales, tramadas en complejas relaciones de poder que usualmente utilizan al género como elemento crucial para las mismas. La formulación de un cosmopolitismo que sortee este problema ha de posibilitar un diálogo cruzando fronteras identitarias, que a su vez promueva creación de redes a través de comunidades feministas que puedan entablar un diálogo público. Habrá que ver en qué condiciones se puede mantener la tensión entre valores universales - tales como los derechos humanos-y experiencias e identidades particulares marginadas. Lo fundamental para que esa tensión sea productiva será, como Reilly (2007) advierte, un compromiso con una reinterpretación crítica de las normas y leyes internacionales; como muchos movimientos feministas de hecho hacen. El objetivo prioritario es hacer servir a las leyes internacionales para proteger las vidas concretas de las mujeres y demás desempoderados ${ }^{5}$.

Como Benhabib (2006) ya explicó en su propuesta de "otro cosmopolitismo" el orden cosmopolita es un orden cosmopolita de leyes. En este

\footnotetext{
${ }^{5}$ Un ejemplo reciente de este compromiso lo constituye la queja que la ONG Women's Link ha interpuesto, con fecha 21 de junio de 2016, ante la Defensora del Pueblo Europeo, con motivo del acuerdo entre la UE y Turquía para desplazar migrantes y refugiados de territorio europeo a Turquía (acuerdo del 18 de marzo de 2016). En la queja denuncian que la Comisión Europea no ha tenido en cuenta ni ha tomado ninguna medida para garantizar el derecho a la dignidad de las personas migrantes y solicitantes de refugio. Tal y como en su página web aclaran (http://www.womenslinkworldwide. org) "es la primera vez que se presenta una queja ante la Defensora del Pueblo Europeo con un análisis diferencial sobre las consecuencias que el Acuerdo tiene para mujeres y niñas y niños refugiados. En la queja se expone que si la Comisión hubiera realizado un análisis del impacto sobre los derechos humanos que tienen sus políticas y acciones, y principalmente el Acuerdo con Turquía, hubiera evitado las dramáticas situaciones que hoy en día están viviendo los miles de personas refugiadas, y en particular las mujeres, niñas y niños, en territorio europeo". Con esta afirmación queda claro en qué sentido la reinterpretación crítica de los derechos humanos tiene enormes potenciales a la hora de encarar retos globales.
} 
orden, y a diferencia de Habermas, las normas no son ni meramente morales ni meramente legales, sino que las normas cosmopolitas constituyen la moralidad misma de la ley. Es la forma como elaboremos esas normas cosmopolitas la que nos lleva a integrar el proceso democrático como un camino de diálogo entre las gentes y las culturas y las instituciones (internacionales, nacionales y locales). En este diálogo pensamos que la voz de la sociedad civil ha de tenerse en cuenta para incorporar la agenda de igualdad de género. Si bien Benhabib no profundiza en el papel de la sociedad civil global sí que nos ofrece un concepto que pensamos que es importante para encontrar ese sujeto universal. Se trata del concepto de "iteraciones democráticas" (2006). Este concepto de Benhabib refiere al proceso dialéctico entre derechos e identidades. En él tanto las identidades como los significados de los derechos son reapropiados y resignificados, tomando así nuevos y diferentes significados. En este sentido, los sujetos participantes en el proceso de diálogo dejan de ser "sujetos dóciles" puesto que se reapropian de los significados, a la vez que pueden alterar su auto-representación y su auto-entendimiento. Esta forma de entender la política y la resignificación de lo universal, especialmente en el marco de los derechos humanos, tiene efectos empoderantes, puesto que el sujeto es protagonista y participante de esa resignificación. Las normas cosmopolitas vistas de esta forma plantean una nueva condición política: lo local, lo nacional y lo global están imbricados (Benhabib, 2006). Las continuas "iteraciones democráticas" pueden contener la promesa de nuevas formas de agencia política, así como de nuevas prácticas cosmopolitas; entre ellas la promesa de lo que aquí llamamos, siguiendo a Reilly (2007), un cosmopolitismo feminista.

\section{Conclusión}

Un cosmopolitismo feminista habrá de tener muy presente los impactos de género que la agenda de globalización neoliberal está causando, a veces con consecuencias silenciadas o incluso peor, interpretadas como promoción de igualdad de género, como hemos visto más arriba en relación a las estrategias del Banco Mundial. El trabajo atento de los grupos feministas de la sociedad civil será esencial para poder profundizar en ese marco de diálogo y reinterpretación continua (Reverter-Bañón 2006, 2013). El cosmopolitismo feminista puede ser un modelo de práctica política emancipatoria, como Reilly (2007) nos propone. Para ello será tan importante y necesario huir de una falsa universalización de los derechos humanos, como entrar en un diálogo que reinterprete críticamente los derechos humanos y descubra su enorme potencial. El cosmopolitismo, sin distinción de sus variables, habrá de ser reflexivo, ya que tiene una orientación ética que va de lo universal a lo particular y de lo particular a lo universal. La 
universalidad, entendida de esta forma, es siempre insuficiente en su alcance, siempre deficiente. Es una universalidad constantemente en suspenso, que sirve como horizonte asintótico a la práctica crítica de una razón inclusiva.

Pensemos que el concepto de "iteraciones democráticas" de Benhabib (2006), del que nos servimos para esta propuesta cosmopolita, alienta la creación de solidaridades más allá de las diferentes fronteras. En este sentido, este cosmopolitismo necesitará de una apertura y ampliación del marco de gobernanza actual (Walby 2002), el cual es muy estrecho para articular el rango de políticas y solidaridades en juego. Podemos decir que hace falta un nuevo concepto de política, que aliado a una gobernanza multilateral permita no sólo la regulación, sino la resistencia y la oposición. Una gobernanza, que podemos llamar participativa y democrática, y que tendrá que abrirse más allá de los actuales límites de lo meramente económico y comercial (Reverter-Bañón 2013, 2017). Y para ello necesitaremos de una incorporación efectiva de la sociedad civil, y en concreto de los movimientos feministas y de mujeres. Una conversación global cosmopolita desde la participación como sujetos que se enuncian como "estratégicamente" universales y que utilice (y reinterprete) el marco global de los derechos humanos podría ser capaz de retar los constreñimientos que la globalización neoliberal, con su superficial discurso de igualdad de género, mantienen. 


\section{Referencias bibliográficas:}

Álvarez, Sonia E. (1998) "El Estado del Movimiento y el Movimiento en el Estado", accesible en el portal Agenda de las Mujeres, http:// agendadelasmujeres.com.ar. Consultado el 4 de junio 2016.

Appiah, Anthony (2006) Ethics in a World of Strangers, New York: Norton.

Barbalet, Jack (2014) "Globalization and cosmopolitanism: Continuity and disjuncture, contemporary and historical", Journal of Sociology, 50(2): 199-212.

Beck, Ulrich (2006) Cosmopolitan Vision, Cambridge Polity Press.

Benería, Lourdes (2012) "The World Bank and gender inequality", Global Social Polic,y 12(2): 175-178.

Benería, Lourdes; Deere, Carmen Diana \& Kabeer, Naila (2012) "Gender and International Migration: Globalization, Development, and Governance", Feminist Economics, 18(2): 1-33.

Benhabib, Seyla (2006) Another Cosmopolitanism, Oxford University Press.

BM Banco Mundial (2011) "Igualdad de Género y Desarrollo. Mensajes principales". Traducción parcial al castellano del texto completo en inglés World Bank (2011) "World Development Report 2012: Gender Equality and Development". Washington, DC: World Bank. Ambos accesibles en: web.worldbank.org/. Consultados el 2 marzo 2016.

Bexell, Magdalena (2012) "Global Governance, Gains and Gender", International Feminist Journal of Politics, 14(3): 389-407.

Brock, Gillian (2013) "Rethinking the Cosmopolitanism versus NonCosmopolitanism Debate: An Introduction", en Brock, Gisela (ed.) Cosmopolitanism versus Non-Cosmopolitanism: Critiques, Defenses, Reconceptualizations, Oxford University Press, 1-34.

Butler, Judith (2015) Notes toward a performative theory of assembly, Harvard University Press.

Caglar, Gülay; Prügl, Elisabeth \& Zwingel, Sussane (2016) "Gender in International Governance", en Steans, J. \& Tepe-Belfrage, D. (eds.) Gender in World Politics, Edward Elgar Publishing, 405-413.

Calkin, Sydney (2015) "Feminism, interrupted? Gender and development in the era of 'Smart Economics"', Progress in Development Studies 15(4): 295-307.

Chant, Sylvia (2012) “The disappearing of 'smart economics'? The World Development Report 2012 on Gender Equality: Some concerns about the preparatory process and the prospects for paradigm change", Global Social Policy 12(2): 198-218.

Crenshaw, Kimberle (1989) "Demarginalizing the Intersection of Race and Sex: A Black Feminist Critique of Antidiscrimination Doctrine, Feminist 
Theory and Antiracist Politics", University of Chicago Legal Forum, 1989(1): 139-169.

Delanty, Gerard (2006) "The cosmopolitan imagination: critical cosmopolitanism and social theory", The British Journal of Sociology, 57(1): 25-47.

Delanty, Gerard (2012a) "The idea of Critical cosmpolitanism”, en Delanty, G. (ed.) Routledge Handbook on Cosmopolitanism Studies, Abingdon, Routledge, 38-46.

Delanty, Gerard (2012b) "The emerging field of cosmopolitanism studies", en Delanty, G. (ed.) Routledge Handbook on Cosmopolitanism Studies, Abingdon, Routledge, 1-8.

Eisenstein, Zillah (1996) Hatreds: Racialized and Sexualized Conficts on the 21st Century. New York: Routledge.

Elias, Juanita (2013) "Davos Woman to the Rescue of Global Capitalism: Postfeminist Politics and Competitiveness Promotion at the World Economic Forum", International Political Sociology, 7: 152-169.

Eyben, Rosalind \& Napier-Moore, Rebecca (2009) "Choosing Words with Care? Shifting meanings of women's empowerment in international development", Third World Quarterly, 30(2): 285-300.

Fraser, Nancy (1989) Unruly practices: power, discourse and gender in contemporary social theory, Cambridge Polity.

Grande, Edgar (2006) "Cosmopolitan political science”. The British Journal of Sociology, 57(1): 87-111.

Habermas, Jürgen (2003) "Towards a Cosmopolitan Europe", Journal of Democracy 14(4): 86-100.

Held, David (2010) Cosmopolitanism: Ideals and Realities, Cambridge: Polity Press.

Hozic, Aida A. \& True, Jacqui (2016) Scandalous Economics: Gender and the Politics of Financial Crises, Oxford University Press.

Hunt, Krista (2002) “The Strategic Co- optation of Women' s Right s. Discourse in the "war on terrorism", International Feminist Journal of Politics, 4(1): 116-121.

Jacquot, Sophie (2003) "Sequences of Policy Change: European Gender Equality Policies and the Emergence of the Gender Mainstreaming Principle, 19891996", ponencia presentada en ECPR Genderal Conference (Marburgo, sep. 2003).

Jaggar, Alison (2000) "Globalizing feminist ethics", en Narayan, Uma, \& Harding, Sandra (eds.) Decentering the center: Philosophy for a multicultural, postcolonial, and feminist world, Bloomington: Indiana University Press, 1-25. 
Kabeer, Naila (2016) “Gender Equality, Economic Growth, and Women's Agency: The 'Endless Variety' and 'Monotonous Similarity' of Patriarchal Constraints", Feminist Economics, 22(1): 295-321.

Kaldor, Mary (2003) "The Idea of Global Civil Society", International Affairs, 79(3): 583-593.

Kantola, Johanna \& Squires, Judith (2012) "From state feminism to market feminism?", International Political Science Review 33: 382-400.

Karamessini, Maria (2014) "Women's Vulnerability to Recession and Austerity", en Karamessini, M \& Rubery, J. (eds.) Women and Austerity. The Economic Crisis and the Future of Gender Equality, Routledge, 4-16. Larner, Wendy (2000) "Neo-liberalism: Policy, ideology, governmentality", Studies in Political Economy 63: 5-25.

Marchetti, Raffaele (2012) "Cosmopolitanism and Global Democratization", en Delanty, G. (ed.) Routledge Handbook on Cosmopolitanism Studies, Abingdon, Routledge, 352-364.

Mendieta, Eduardo (2009) "From imperial to dialogical cosmopolitanism", Ethics \& Global Politics, 2(3): 241-258.

Mignolo, Walter (2000) "The Many Faces of Cosmo-polis: Border Thinking and Critical Cosmopolitanism", Public Culture, 12(3): 721-748.

Milanovic, Branco (2016) Global Inequality. A new approach for the age of globalization, Cambridge, Massachusetts: Harvard University Press.

Mohanty, Chandra Talpade (1984) "Under Western Eyes: Feminist Scholarship and Colonial Discourses”, boundary 2, 12(3): 333-358.

Mohanty, Chandra Talpade (2003) Feminism Without Borders: Decolonizing Theory, Practicing Solidarity, Duke University Press Books

Mohanty, Chandra Talpade (2013). "Transnational Feminist Crossings: On Neoliberalism and Radical Critique", Signs: Journal of Women in Culture \& Society, 38 (4): 967.

Molyneux, Maxine (2006) "Mothers at the Service of the new poverty agenda: PROGRESA/Oportunidades, Mexico's Conditional Transfer Programme”, Journal of Social Policy and Administration, 40(4): 425-449.

Molyneux, Maxine (2007) "Change and Continuity in Social Protection in Latin America: Mothers at the Service of the State?" Gender and Development Paper No. 1. Geneva: UNRISD. Accesible: www.unrisd.org.

Nussbaum, Martha (1997) “Kant and Cosmopolitanism”, en Bohman, J. \& LutzBachman, L. (eds.) Pertpetual Peace: Essays on Kant's Cosmopolitan Ideal, Cambridge, MIT Press, 25-57.

Okin, Susan Moller (1999) Is multiculturalism bad for women?, Princeton University Press. 
Okin, Susan Moller (2000) "Feminism, women's human rights, and cultural differences", en Narayan, Uma, \& Harding, Sandra (eds.) Decentering the center: Philosophy for a multicultural, postcolonial, and feminist world, Bloomington: Indiana University Press, 26-46.

Orloff, Ann Shola \& Shiff, Talia (2016) "Feminism/s in Power: Rethinking Gender Equality after the Second Wave", Perverse Politics? Feminism, Anti-Imperialism, Multiplicity. 31: 109-134.

Pieri, Elisa (2014) “Contested Cosmopolitanism”, en L. Kaunonen (Ed.), Cosmopolitanism and Transnationalism: Visions, Ethics, Practices: COLLeGIUM: Studies across Disciplines in the Humanities and Social Sciences, 15: 14-38. Helsinki: Helsinki Collegium for Advanced Studies.

Potrafke, Niklas (2015) “The Evidence on Globalisation”, The World Economy, 38(3): 509-552.

Prügl, Elisabeth (2017) "Neoliberalism with a Feminist Face: Crafting a new Hegemony at the World Bank", Feminist Economics, 23(1): 30-53.

Prügl, Elisabeth \& True, Jaqui (2014) "Equality means businee? Governing Gender through transnational public-private partnerships", Review of International Political Economy, 21(6): 1137-1169.

Rai, Shirin M. \& Waylen, Georgina eds. (2008) Global Governance. Feminist Perspectives, Palgrave Macmillan.

Razavi, Shahra (2012) "WDR 2012: Too little, and already a bit out-of-date?", Global Social Policy, 12(2): 193-197.

Reilly, Niamh (2007) "Cosmopolitan Feminism and Human Rights", Hypatia 22(4): 180-198.

Reverter-Bañón, Sonia (2006) Civil Society and Gender Equality: A Theoretical Approach, Civil Society Series. Centre for Civil Society. London School of Economics and Political Sciences.

Reverter-Bañón, Sonia (2013) “Género y gobernanza”, en González-Esteban, Elsa (ed.) Ética y Gobernanza. Un cosmopolitismo para el siglo XXI, Granada: Comares. Colección Filosofía Hoy, 103-122.

Reverter-Bañón, Sonia (2017) "Performatividad: la teoría especial y la general”, Isegoría. Revista de filosofía moral y política, (en prensa).

Sahlins, Marshall (2000) Culture in practice. Selected Essays, New York: Zed Books.

Sorensen, Asger (2016) 'Cosmopolitanism - Not a 'major ideology', but still an ideology", Philosophy and Social Criticism, 42(2): 200-224.

Stern, Maria (2016) "Postestructuralist feminism in world politics", en Steans, J. \& Tepe-Belfrage, D. (eds.) Gender in World Politics, Edward Elgar Publishing, 33-40.

Tronto, Joan C. (2013) Caring Democracy. Markets, Equality and Justice, New York \& London: New York University Press. 
Walby, Sylvia (2002) "Feminism in a global era", Economy and Society, 31(4): 533-557.

Waylen, Georgina (2008), "Gendering Governance"e, en Goertz, G. y Mazur, A. (eds.), Politics, Gender and Concepts: Theory and Methodology. Cambridge University Press, 114-35.

Wilson, Kalpana (2015) "Towards a Radical Re-appropriation: Gender, Development and Neoliberal Feminism", Development and Change 46(4): 803-832.

World Bank (2008) "Gender equality as smart economics: World Bank group Gender Action Plan. First year progress report (Jan 2007-Jan 2008)”. Washington, DC: World Bank. Accesible en: web.worldbank.org/ Consultado el 2 de marzo 2016.

World Bank (2011) "World Development Report 2012: Gender Equality and Development". Washington, DC: World Bank. Accesible en: web. worldbank.org/ Consultado el 2 de marzo 2016.

Zuckerman, Elaine (2007) Gender Equality as Smart Economics: A World Bank Group Gender Action Plan (GAP) (Fiscal years 2007-10): A Critique, Mimeo. Washington, DC: GenderAction. Accesible en www. genderaction.org. Consultado el 8 abril 2016.

Zuckerman, Elaine \& Qing, Wu (2003) Reforming the World Bank: Will the New Gender Strategy Make a Difference? A Study With China Case Examples, Washington, DC: Heinrich Boll Foundation/Gender Action. Accesible en www.genderaction.org. Consultado el 2 de marzo 2016. 
